\title{
PERFIL HEMATOLÓGICO DE CÃES E GATOS NA CIDADE DE BENTO GONÇALVES, RIO GRANDE DO SUL, BRASIL
}

\author{
Shimena Tesser \\ Natália Inês Cavagnolli ${ }^{1}$ \\ Tânia Torriani² \\ Adriana Dalpicolli Rodrigues ${ }^{3}$
}

TESSER, S.; CAVAGNOLLI, N. I.; TORRIANI, T.; RODRIGUES, A. D. Perfil hematológico de cães e gatos na cidade de Bento Gonçalves, Rio Grande do Sul, Brasil. Arq. Ciênc. Vet. Zool. UNIPAR, Umuarama, v. 19, n. 1, p. 47-51, jan./mar. 2016.

RESUMO: O perfil hematológico é importante na clínica de cães e gatos para auxílio na busca diagnóstica, prognóstica e monitoramento da saúde ou terapia animal. Essa avaliação possibilita ao médico veterinário verificar a presença de: anemia, doenças inflamatórias/infecciosas e neoplásicas em animais muitas vezes assintomáticos. Em vista disso, objetivou-se efetuar um levantamento de resultados de exames hematológicos (hemograma) realizados em cães e gatos, em um laboratório veterinário na cidade de Bento Gonçalves (RS), no período de 2010 a 2013. Foram analisados 2.864 hemogramas (2.425 de cães; 439 de gatos). Observou-se resultados diminuídos para eritrócitos (27\%), hemoglobina (24,7\%) e hematócrito (27,1\%), especialmente, em cães machos. Os leucócitos apresentaram-se aumentados em torno de 30,5\% dos cães e 42,1\% dos gatos, de ambos os sexos. O número de plaquetas mostrou-se baixo, principalmente, nos gatos (58,8\%). Verificou-se que entre cães e gatos há uma variação importante nos exames hematológicos por serem espécies diferentes. Entretanto, quando comparados os resultados por sexo da mesma espécie não houve diferença significativa. Mais estudos sobre o hemograma desses animais se fazem necessários, principalmente relacionando o estado de saúde e suas condições de vida, favorecendo a um diagnóstico clínico fidedigno.

PALAVRAS-CHAVE: Animais domésticos. Diagnóstico. Hemograma.

\section{HEMATOLOGICAL PROFILE OF DOGS AND CATS IN THE CITY OF BENTO GONÇALVES, RIO GRANDE DO SUL, BRAZIL}

\begin{abstract}
The hematological profile is clinically important for dogs and cats to aid in the prognostic and monitoring of the animal's health or therapy. This assessment enables the veterinarian to check for anemia, inflammatory/infectious and neoplastic diseases in animals which are often asymptomatic. In light of this, the purpose of this study was to perform a survey of hematological test results (blood count) performed in dogs and cats in a veterinary laboratory in the city of Bento Gonçalves (RS) between 2010 and 2013. A total 2.864 blood counts (2.425 dogs and 439 cats) were analyzed. The observed results were reduced for erythrocytes $(27 \%)$, hemoglobin $(24.7 \%)$ and hematocrit $(27.1 \%)$, especially in male dogs. Elevated percentages of leukocytes were observed in $\operatorname{dogs}(30.5 \%)$ and cats $(42.1 \%)$ of both sexes. The number of platelets was shown to be lower, mainly in cats $(58.8 \%)$. It was found that among dogs and cats, there is a significant variation in hematological tests since they are different species. However, when comparing the results by the sex of the same species, there were no significant difference. Further studies on blood count of animals are needed, particularly relating to health and their living conditions, favoring a reliable clinical diagnosis.
\end{abstract}

KEYWORDS: Blood count. Diagnostic. Domestics animals.

\section{PERFIL HEMATOLÓGICO DE PERROS Y GATOS EN LA CIUDAD DE BENTO GONÇALVES, RIO GRANDE DO SUL, BRASIL}

RESUMEN: El perfil hematológico es importante en la clínica de perros y gatos para ayudar en la búsqueda de diagnóstico, pronóstico y seguimiento de la salud o terapia animal. Esa evaluación permite al médico veterinario verificar la presencia de: anemia, enfermedades inflamatorias / infecciosas y neoplásicas en animales a menudo asintomáticos. Así, el objetivo ha sido llevar a cabo un estudio de los resultados de las pruebas hematológicas (hemograma), realizadas en perros y gatos, en un laboratorio veterinario de la ciudad de Bento Gonçalves (RS), en el período de 2010 a 2013. Se analizaron 2864 hemogramas (2.425 de perros y 439 de gatos). Se observó resultados reducidos para eritrocitos (27\%), hemoglobina (24,7\%) y hematocrito $(27,1 \%)$, especialmente en perros machos. Los leucocitos se presentaron aumentados alrededor del $30,5 \%$ de los perros y el $42,1 \%$ de los gatos, de ambos sexos. El número de plaquetas se mostró inferior, especialmente en los gatos (58,8\%). Se encontró que entre perros y gatos hay una variación significativa en los exámenes hematológicos, pues son especies diferentes. Sin embargo, al comparar los resultados por sexo de la misma especie no hubo diferencia significativa. Más estudios sobre el

DOI: https://doi.org/10.25110/arqvet.v19i1.2016.5790

${ }^{1}$ Bacharel em Biomedicina pela Faculdade da Serra Gaúcha.

${ }^{2}$ Bacharel em Biomedicina e Especialista em Citologia Diagnóstica pela Universidade Feevale. Docente do Curso de Biomedicina da Faculdade da Serra Gaúcha, Proprietária do Laboratório Veterinário Biovet

${ }^{3}$ Bacharel em Biomedicina pela Universidade Feevale. Mestre em Biotecnologia pela Universidade de Caxias do Sul. Analista científica no Laboratório Alfa LTDA. 
hemograma de esos animales se hacen necesarios, principalmente relacionando el estado de salud y sus condiciones de vida, lo que favorece un diagnóstico clínico fiable.

PALABRAS CLAVE: Animales domésticos. Diagnóstico. Hemograma.

\section{Introdução}

O perfil hematológico é importante na clínica de animais domésticos como cães e gatos, pois auxilia no estabelecimento de diagnósticos, prognósticos e acompanhamento da saúde ou terapia do animal (GONZÁLEZ; FÉLIX; SANTOS, 2005). O hemograma, de modo isolado, é um recurso de diagnóstico limitado na maioria dos casos (execto para avaliação de hemoparasitas), mas é possível estabelecer um ponto de partida para o diagnóstico rápido e preciso do paciente. Através desse exame, o médico veterinário obtém informações sobre o estado clínico geral do animal, situações deficitárias (anemias), policitemia, processos infecciosos bacterianos, virais ou parasitológicos, alterações plaquetárias, neoplasias, doenças degenerativas, entre outros, até mesmo em pacientes assintomáticos (GONZÁLEZ; FÉLIX; SANTOS, 2005; GONZÁLEZ; SILVA, 2006; ZAVALA, 2007).

A interpretação do hemograma é delicada, devido a variações que ocorrem de acordo com a raça, idade, alimentação, manejo, condições geográficas e habitacionais, e estado fisiológico (lactação, gestação, estado reprodutivo) do animal (GONZÁLEZ; SCHEFFER, 2003). Além disso, determinadas situações como: medo, excitação ou estresse desencadeiam respostas hormonais que alteram a concentração das células sanguíneas. A coleta sanguínea é um exemplo de uma situação de estresse aguda que pode desencadear diminuição da concentração de hemoglobina corpuscular média, aumento do número total de eritrócitos, hematócrito, plaquetas e de leucócitos (totais, neutrofilia, eosinofilia, linfocitose). Os leucócitos, em particular, sofrem mais alterações nos gatos (até quatro vezes mais) do que nos cães (FAM et al., 2010).

De modo geral, para uma melhor interpretação dos exames laboratoriais e um correto diagnóstico clínico, é indispensável contar com valores de referência apropriados para cada população de animais (BOURGÈS-ABELLAet al., 2015). Entretanto, sabe-se que a maioria dos valores de referência se encontram na literatura estrangeira (GONZÁLEZ et al., 2001; ZAVALA, 2007) e costumam ser usados referente a grupos de animais similares (GONZÁLEZ; SCHEFFER, 2003), dificultando a exatidão nas interpretações. Em vista disso, o presente estudo realizou um levantamento do perfil hematológico de cães e gatos em um laboratório de análises clínicas veterinárias na cidade de Bento Gonçalves, Rio Grande do Sul, Brasil, no período de 2010 a 2013.

\section{Material e métodos}

Foi realizado um estudo transversal e retrospectivo, por meio de consulta em banco de dados de resultados do perfil hematológico de cães e gatos realizados em um laboratório de análises clínicas veterinárias da cidade de Bento Gonçalves - Rio Grande do Sul, Brasil. Foram avaliados resultados de dosagem de eritrócitos, hemoglobina, hematócrito, contagem total e diferencial de leucócitos e contagem de plaquetas, efetuados neste laboratório, entre junho de 2010 e dezembro de 2013.

No laboratório as análises hematológicas eram realizadas em aparelho automatizado Sysmex Iv Poch 100, específico para uso veterinário, calibrados com referências de cada parâmetro do teste para animais, de acordo com as normas do fabricante. Os valores de referências utilizados como base para interpretação das alterações dos exames foram padronizados pelo Lacvet - Laboratório de Análises Clínicas Veterinária, Universidade Federal do Rio Grande do Sul (descritos na Tabela 1).

Os dados foram apresentados em valores mínimos e máximos, frequências e percentuais absolutos de cada exame analisado. Para a comparação entre sexos de cada espécie, foi aplicado o teste de qui-quadrado, tabulações cruzadas, considerando-se significativo para $\mathrm{p} \leq 0,05$, no programa SPSS 20.0 para Windows.

Tabela 1: Valores de Referência (VR) padronizados pela Lacvet-UFRGS.

\begin{tabular}{ccc}
\hline EXAMES & VR CÃES & VR GATOS \\
\hline ERI & $5,5-8,5.10 \% / \mathrm{uL}$ & $5-10.10^{6} / \mathrm{uL}$ \\
HGB & $12-18 \mathrm{~g} / \mathrm{dL}$ & $8-15 \mathrm{~g} / \mathrm{dL}$ \\
HCT & $37-55 \%$ & $24-45 \%$ \\
WBC & $6.000-17.000 \mathrm{uL}$ & $5.000-19.500 \mathrm{uL}$ \\
NB & $0-3 \%$ & $0-3 \%$ \\
NS & $60-77 \%$ & $35-75 \%$ \\
L & $12-30 \%$ & $20-55 \%$ \\
M & $3-10 \%$ & $1-4 \%$ \\
E & $2-10 \%$ & $2-12 \%$ \\
PLT & $200-500.10^{3} / \mathrm{uL}$ & $300-800.10^{3} / \mathrm{uL}$
\end{tabular}

Siglas: Eritrócitos (ERI); Hemoglobina (HGB); Hematócrito (HCT); Leucócitos Totais (WBC); Neutrófilo Bastonetes (NB); Neutrófilos Segmentados (NS); Linfócitos (L); Monócitos (M); Eosinófilos (E) e Plaquetas (PLT).

\section{Resultados}

Foram analisados 2.864 resultados de exames hematológicos, sendo 2.425 cães (1.073 machos e 1.352 fêmeas) e 439 gatos (242 machos e 197 fêmeas). Os resultados dos hemogramas estão apresentados na Tabela 2, pode-se observar que há valores mínimos e máximos discrepantes entre os animais estudados em cada análise.

A série vermelha, caracterizada por eritrócitos, hemoglobina e hematócrito, apresentou valores abaixo do valor de referência, em porcentagens maiores, em cães machos $(27 \%, 24,7 \%$ e $27,1 \%$, respectivamente). O hematócrito apresentou-se elevado em um maior número de gatos (machos: $12,4 \%$ e fêmeas: $16,2 \%$ ). Na série branca, as principais alterações observadas foram o número aumentado de leucócitos em $30,7 \%$ e $30,2 \%$ dos cães machos e fêmeas, respectivamente, e 40,5\% dos gatos machos e 44,2\% das fêmeas. No diferencial dos leucócitos, os neutrófilos foram as células que apresentaram importante aumento em todos os animais. $\mathrm{O}$ número de plaquetas mostrou-se com valores abaixo da referência principalmente para os gatos (machos: $60,7 \%$ e 
fêmeas: $56,4 \%$ ). Não foram observadas diferenças estatísticas entre os resultados hematológicos de animais machos e fêmeas da mesma espécie (Tabela 2).
Tabela 2: Frequências de exames hematológicos abaixo, dentro ou acima da normalidade em cães e gatos, por sexo, avaliados em um laboratório de análises clínicas veterinárias de Bento Gonçalves-RS.

\begin{tabular}{|c|c|c|c|c|c|c|}
\hline \multirow[t]{2}{*}{ EXAMES } & \multicolumn{2}{|c|}{ CÃES } & \multicolumn{4}{|c|}{ GATOS } \\
\hline & $\begin{array}{l}\text { Macho } \\
\%(n)\end{array}$ & $\begin{array}{l}\text { Fêmea } \\
\%(n)\end{array}$ & $\begin{array}{c}\mathrm{p}- \\
\text { valores } *\end{array}$ & $\begin{array}{l}\text { Macho } \\
\%(n)\end{array}$ & $\begin{array}{l}\text { Fêmea } \\
\%(n)\end{array}$ & $\begin{array}{c}\mathrm{p}- \\
\text { valores } *\end{array}$ \\
\hline ERI [mín -máx] & {$[1-9,7]$} & {$[0,5-56,4]$} & & {$[1-10,35]$} & {$[1,4-10]$} & \\
\hline ABAIXO & $27(290)$ & $0,1(1)$ & 0,571 & $15,3(37)$ & $11,7(23)$ & 0,358 \\
\hline NORMAL & $72,2(775)$ & $98,9(1338)$ & & $84,3(204)$ & $88,3(174)$ & \\
\hline ACIMA & $0,8(8)$ & $1(13)$ & & $0,4(1)$ & - & \\
\hline HGB [mín -máx] & {$[2,9-60]$} & {$[1-23]$} & & {$[1-10,5]$} & {$[2,6-18]$} & \\
\hline ABAIXO & $24,7(265)$ & $20,9(283)$ & 0,073 & $15,3(37)$ & $11,2(22)$ & 0,327 \\
\hline NORMAL & $67,5(724)$ & $71,5(967)$ & & 76,9 (186) & $82,2(162)$ & \\
\hline ACIMA & $7,8(84)$ & $7,6(102)$ & & $7,8(19)$ & $6,6(13)$ & \\
\hline HCT [mín -máx] & {$[8,4-65,8]$} & {$[5-61,8]$} & & [6-56] & {$[8,5-55]$} & \\
\hline ABAIXO & $27,1(291)$ & $23,2(314)$ & 0,088 & $15,7(38)$ & $10,2(20)$ & 0,156 \\
\hline NORMAL & $66,7(716)$ & $70,4(952)$ & & $71,9(174)$ & $73,6(145)$ & \\
\hline ACIMA & $6,2(66)$ & $6,4(86)$ & & $12,4(30)$ & $16,2(32)$ & \\
\hline WBC [mín -máx] & [1700-170000] & [880-455000] & & [3000-900000] & [1400-170000] & \\
\hline ABAIXO & $4,6(49)$ & $3,6(48)$ & 0,405 & $2,9(7)$ & $3,5(7)$ & 0,648 \\
\hline NORMAL & 64,7 (694) & $66,2(895)$ & & $56,6(137)$ & $52,3(103)$ & \\
\hline ACIMA & $30,7(330)$ & $30,2(409)$ & & $40,5(98)$ & $44,2(87)$ & \\
\hline NB [mín-máx] & [0-39] & {$[0-37]$} & & {$[0-15]$} & {$[0-18]$} & \\
\hline ABAIXO & - & - & 0,069 & - & - & 0,719 \\
\hline NORMAL & $82,8(888)$ & $86,1(1164)$ & & $90,9(220)$ & $91,9(181)$ & \\
\hline ACIMA & $17,2(185)$ & $13,9(188)$ & & $9,1(22)$ & $8,1(16)$ & \\
\hline NS [mín-máx] & [7-97] & [0-99] & & [10-97] & [9-97] & \\
\hline ABAIXO & $15,1(162)$ & 14,3 (193) & 0,833 & $3,3(8)$ & $3,1(6)$ & 0,322 \\
\hline NORMAL & $47,2(506)$ & 48,0 (649) & & $54,1(131)$ & $47,2(93)$ & \\
\hline ACIMA & $37,7(405)$ & $37,7(510)$ & & $42,6(103)$ & $49,7(98)$ & \\
\hline L [mín-máx] & {$[1-87]$} & {$[1-100]$} & & {$[1-88]$} & {$[1-89]$} & \\
\hline ABAIXO & $25,3(272)$ & $27,3(369)$ & 0,551 & $50,8(123)$ & $56,8(112)$ & 0,151 \\
\hline NORMAL & $60,8(652)$ & $58,9(797)$ & & 46,7 (113) & $38,6(76)$ & \\
\hline ACIMA & 13,9 (149) & $13,8(186)$ & & $2,5(6)$ & $4,6(9)$ & \\
\hline M [mín -máx] & {$[0-54]$} & [0-71] & & [0-14] & {$[0-25]$} & \\
\hline ABAIXO & $45,5(488)$ & $43,1(583)$ & 0,452 & $2,1(5)$ & $1(2)$ & 0,603 \\
\hline NORMAL & $51,7(555)$ & $54,3(734)$ & & 88 (213) & 90,4 (178) & \\
\hline ACIMA & $2,8(30)$ & $2,6(35)$ & & $9,9(24)$ & $8,6(17)$ & \\
\hline E [mín-máx] & [0-44] & [0-67] & & [0-25] & [0-29] & \\
\hline ABAIXO & $42,3(454)$ & $41(554)$ & 0,682 & $31,8(77)$ & $33(65)$ & 0,752 \\
\hline NORMAL & $52,8(567)$ & $53,5(724)$ & & $60,3(146)$ & $60,9(120)$ & \\
\hline ACIMA & $4,9(52)$ & $5,5(74)$ & & 7,9 (19) & $6,1(12)$ & \\
\hline PLT [mín -máx] & [5000-944000] & [7000-1141000] & & [110000-900000] & [23000-900000] & \\
\hline ABAIXO & $24,5(263)$ & $20,4(275)$ & 0,860 & $60,7(147)$ & $56,4(111)$ & 0,393 \\
\hline NORMAL & $66,9(718)$ & $67,5(913)$ & & $38,9(94)$ & $42,1(83)$ & \\
\hline ACIMA & $8,6(92)$ & $12,1(164)$ & & $0,4(1)$ & $1,5(3)$ & \\
\hline
\end{tabular}

Siglas: Eritrócitos (ERI); Hemoglobina (HGB); Hematócrito (HCT); Leucócitos Totais (WBC); Neutrófilo Bastonetes (NB); Neutrófilos Segmentados (NS); Linfócitos (L); Monócitos (M); Eosinófilos (E) e Plaquetas (PLT). *Relação entre machos e fêmeas - teste de qui-quadrado, tabulações cruzadas, com $p<0,05$.

\section{Discussão}

As mudanças na sociedade decorrentes da urbanização vêm destacando a proximidade entre os humanos e os animais de estimação, devido os benefícios que os mesmos causam (STALLIVIERI et al., 2013). Embora ainda seja rara a realização de exames rotineiros em animais domésticos, os tutores se preocupam cada vez mais com a saúde e bem estar animal. O número de pesquisas de parâmetros laboratoriais de cães e gatos com estado fisiológico normal são escassos
(RORTVEIT et al., 2015), pois normalmente os exames são realizados com eles apresentando alguma enfermidade. No presente estudo, foram analisados resultados hematológicos de cães e gatos em rotina clínica (incluindo, possivelmente, doentes) num período de três anos, onde verificou-se que haviam mais dados para cães, talvez por ainda ser o animal que mais convive com o homem (STALLIVIERI et al., 2013).

Em um estudo realizado por Fernandes et al. (2008), foi observado que, dos 59 cães soropositivos para o hemoparasita Ehrlichia canis, 100\% apresentaram alterações he- 
matológicas, onde se destacam anemia (66\%), leucopenia $(42 \%)$ e trombocitopenia $(87 \%)$. Os percentuais referentes à diminuição nos parâmetros hematológicos são mais altos do que os encontrados no presente estudo, entretanto, não se têm o conhecimento da presença desse hemoparasita nos animais avaliados.

No presente estudo não foi possível obter informações sobre a raça dos animais, mas sabe-se que entre eles existem errantes, onde $24,5 \%$ dos cães apresentaram valores abaixo dos valores de referência para eritrócitos, hemoglobina e hematócrito, corroborando com os dados encontrados por Basílio et al. (2010). Esses autores realizaram um estudo determinando o perfil hematológico, transaminase pirúvica e creatinina em 33 cães errantes pré-cirúrgicos do Paraná, onde $21,2 \%$ apresentaram baixos valores de eritrograma, sendo que $66,6 \%$ apresentaram eosinofilia, no entanto, os exames bioquímicos não mostraram alterações significativas. As alterações no eritrograma sugerem anemia hemolítica decorrentes de hemoparasita ou anemia hipoplásica, por deficiência proteica, mineral e/ou vitamínicas (má nutrição) (Basílio et al., 2010).

Em Sobral, Ceará, Leite et al. (2013), analisaram um relato de caso de um cão da raça Cocker Spaniel, macho, com cinco anos de idade, diagnosticado com leptostirose. Este foi submetido a exames hematológicos, os quais eritrócitos, hemoglobina, hematócrito e plaquetas apresentaram valores diminuídos comparados aos valores de referência sugeridos pelo autor, e leucócitos aumentados, com destaque para neutrófilos bastonetes e segmentados. No presente estudo, a média de cães machos com todos estes parâmetros alterados, da mesma forma que o estudo citado, é de aproximadamente $28 \%$.

Estudo realizado por Martins et al. (2012), avaliaram o perfil hematológico de cães obesos e senis em uma cidade de São Paulo. O grupo estudado continha 59 cães, sendo 30 com idade entre dois e oito anos (grupo 1) e 29 acima de oito anos (grupo 2) separados em fêmeas e machos. Aumento de eritrócitos e hemoglobina foram relatados em $23,3 \%$ e 46,7\%, respectivamente, dos animais do grupo 1 e $27,59 \%$ e $41,37 \%$ do grupo 2 . O número de NB demonstrou-se acima do valor de referência em $90 \%$ dos animais do grupo 1 e 68,96\% do grupo 2. Os eosinófilos mostraram-se aumentados em $34,48 \%$ dos cães do grupo 2 , sendo que em machos a média foi superior à média das fêmeas (2260 \pm 1363 / $\mu \mathrm{L}$ e $758 \pm 427 / \mu \mathrm{L}$, respectivamente). No presente estudo, o aumento nesses parâmetros atingiu um percentual bem menor de animais (Tabela 2) do que relatado por Martins et al. (2012).

Costa et al. (2008) verificaram o perfil hematológico de gatos mestiços (sem raça definida) e persas, e evidenciaram que há diferença estatística significativa entre as raças referentes ao número de basófilos, estando em maior número em gatos persas. No presente estudo foi encontrado valores iguais ou menor que 1 em relação aos basófilos, os quais não foram incluídos na Tabela 2 por não terem significado clínico. Costa et al. (2008) ressalta ainda que gatos machos e fêmeas diferem-se pouco em valores de referência e a atenção deve ser voltada para a raça. O presente estudo confirma que não há diferença estatística entre os sexos tanto para gatos quanto para cães.

Monteiro et al. (2011) realizaram um ensaio sobre os efeitos do glicocorticoide prednisona em oito gatos na cidade de Recife, e observaram que após a administração do fármaco, houve um aumento plaquetário (média de 519.3 $2228,2 \times 10^{3}$ / $\mu \mathrm{L})$, como também monocitose $\left(1,94 \pm 1.458,5 \times 10^{3} / \mu \mathrm{L}\right)$, não havendo alteração significativa na série vermelha. No presente estudo não se tem a informação sobre a utilização ou não de qualquer tipo de fármaco administrado nos animais, mas $10,5 \%$ dos cães e $0,9 \%$ dos gatos apresentam plaquetas aumentadas. A diminuição de plaquetas também foi relevante, especialmente nos gatos (60,7\% machos e 56,4\% fêmeas). Nesses animais é comum a presença de agregados plaquetários que interferem na análise, muitas vezes esta alteração ocorre devido a falhas na coleta causada principalmente pela agitação do animal (HLAVAC, 2010).

Para cada parâmetro analisado neste estudo, há uma suspeita clínica relacionada as alterações que podem ser encontradas. O eritrograma pode caracterizar um diagnóstico específico, que é o caso das anemias, porém também sofre discreta variação com a idade do animal; já o leucograma pode indicar patologias, como por exemplo, aumento no número de NS, encontrada em 37,7\% de cães (machos e fêmeas), $42,6 \%$ e $49,7 \%$ de gatos machos e fêmeas, respectivamente. No presente o estudo, pode ser indicativo de inflamação (por infecção, imune ou necrose) e os eosinófilos podem indicar processos de hipersensibilidade e parasitismo (BIONDO, 2005).

Além das patologias, também podem ocorrer alterações nos parâmetros metabólicos em decorrência da idade, raça, alimentação, habitat, o estado psicológico do animal (estresse, medo, excitação) e estado fisiológico (cio, gestação e lactação) (GONZÁLEZ; SCHEFFER, 2003; FAM et al., 2010). Cabe salientar que a coleta de sangue pode causar um alto nível de estresse, por isso, as fases pré-analítica, analítica e pós-analítica de qualquer análise laboratorial devem ser cuidadosamente efetuadas.

\section{Conclusão}

Os resultados do perfil hematológico de cães e gatos atendidos em um laboratório veterinário em Bento Gonçalvez - Rio Grande do Sul - Brasil, mostraram uma grande variação nos valores encontrados entre as espécies, porém entre os sexos a variação é pequena e não tem diferença estatística.

A realização de dosagens hematológicas são de extrema importância para avaliar e acompanhar o estado de saúde de cães e gatos. Mais estudos se fazem necessários na área de análises clínicas veterinárias em diferentes grupos de animais e em relação à determinação de valores de referências próprios para cada raça, faixa etária e região, considerando informações sobre as condições de vida do animal. Esses fatores elucidariam melhor as análises e principalmente auxiliariam em uma adequada interpretação dos resultados e um diagnóstico fidedigno.

\section{Referências}

BASÍLIO, G. et al. Análises hematológicas e bioquímicas séricas de cães errantes da região de Guarapuava - PR. In: ENCONTRO ANUAL DE INICIAÇÃO CIENTÍFICA, 19., 2010, Guarapuava. Anais... Guarapuava: UNICENTRO, 2010 . 
BIONDO, A.W. Interpretação do leucograma. In: SIMPÓSIO DE PATOLOGIA CLÍNICA VETERINÁRIA DA REGIÃO SUL DO BRASIL, 2., 2005, Porto Alegre. Anais... Porto Alegre: UFRGS, 2005. p. 29-35.

BOURGÈS-ABELLA, N. H. et al. Reference intervals, intraindividual and interindividual variability, and reference change values for hematologic variables in laboratory beagles. Journal of the American Association for Laboratory Animal Science, v. 54, n. 1, p. 17-24, 2015.

COSTA, A. S. Perfil hematológico e bioquímico sérico de gatos domesticos (Felis catus- Linnaeus, 1758) da raça persa e mestiços. Uberlândia, 2008. Dissertação (Mestrado em Ciências Veterinárias) - Universidade Federal de Uberlândia, Uberlândia, 2008.

FAM, A. L. P. D. et al. Alterações no leucograma de felinos domésticos (Felis catus) decorrentes de estresse agudo e crônico. Revista Acadêmica: Ciências Agrárias e Ambientais, v. 8, p. 299-306, 2010.

FERNANDES, P. V. B. et al. Alterações hematológicas e bioquímicas em cães soropositivos para Erlichia canis no período de 2002 a 2008. In: CONGRESSO BRASILEIRO DE MEDICINA VETERINÁRIA, 35., 2008, Gramado. Anais... Gramado: Sovergs, 2008.

GONZÁLEZ, F. H. D. et al. Perfil bioquímico sanguíneo de cães e gatos na cidade de Porto Alegre, Rio Grande do Sul, Brasil. Arquivos da Faculdade de Veterinária UFRGS, v. 29, p. 1-6, 2001.

GONZÁLEZ, F. H. D.; SCHEFFER, J. F. Perfil Sanguíneo: ferramenta de análise clínica, metabólica e nutricional. In: SIMPÓSIO DE PATOLOGIA CLÍNICA VETERINÁRIA DA REGIÃO SUL DO BRASIL, 1., 2003, Porto Alegre. Anais... Porto Alegre: UFRGS, 2003. p. 73-89.

GONZÁLEZ, F. H. D.; SANTOS, A. P. Patologia clínica veterinária. In: SIMPÓSIO DE PATOLOGIA CLÍNICA VETERINÁRIA DA REGIÃO SUL DO BRASIL, 2., 2005, Porto Alegre. Anais... Porto Alegre: UFRGS, 2005, p. 91.

GONZÁLEZ, F. H. D.; SILVA, S. C. Introdução a bioquímica veterinária. Porto Alegre: UFRGS, 2006.

HLAVAC, N. R. C. Avaliação de parâmetros plaquetários em cães saudáveis: efeitos da temperatura, tempo e tipo de anticoagulante. 2010. Dissertação (Mestrado em Ciências Veterinárias) - Faculdade de Veterinária, Universidade Federal do Rio Grande do Sul, Porto Alegre, 2010 .

LACVET - Laboratório de Análises Clínicas Veterinária, Universidade Federal do Rio Grande do Sul (UFRGS). Valores de referência. Disponível em: <http:/www.ufrgs. br/lacvet/tabela_referencia.php>. Acesso em: 20 ago. 2015.

MARTINS, C. R. Perfil hematológico e bioquímico de cães (Canis familiaris) obesos e idosos. Uberlândia.
2012. Dissertação (Mestrado em Ciências Veterinárias) Faculdade de Medicina Veterinária, Universidade Federal de Uberlândia, Uberlândia , 2012.

MONTEIRO, J. N. M. et al. Hematologia, bioquímica e cortisol de gatos tratados com prednisolona. Medicina Veterinária-Recife, v. 5, p. 14-18, 2011.

RORTVEIT, R. et al. Age-related changes in hematologic and serum biochemical variables in dogs aged 16-60 days. Veterinary Clinical Pathology, v. 44, n. 1, p. 47-57, 2015.

STALLIVIERI, F. M. et al. Helmintos intestinais em cães domiciliados e aspectos socioeconômicos e culturais das famílias proprietárias dos animais de Lages, SC, Brasil.

Archives of Veterinary Science, v. 18, n. 3, p. 22-27, 2013.

ZAVALA, M.V. G. Aspectos clínicos e laboratoriais de cães suspeitos de infecções causadas por Erlichia spp. e Anaplasma spp. em Campos dos Goytacazes, RJ. 2007. Dissertação (Mestrado) - Centro de Ciências e Tecnologias Agropecuárias - Universidade Estadual do Norte

Fluminense Darcy Ribeiro, Campos dos Goytacazes, 2007.

Recebido em: 27.08.2015 Aceito em: 02.03.2016 